\title{
Screening Cherry Germ Plasm for Resistance to Leaf Spot
}

\author{
Phillip S. Wharton, Department of Plant Pathology, Amy Iezzoni, Department of Horticulture, and Alan L. Jones, \\ Department of Plant Pathology, Michigan State University, East Lansing 48824
}

\begin{abstract}
Wharton, P. S., Iezzoni, A., and Jones, A. L. 2003. Screening cherry germ plasm for resistance to leaf spot. Plant Dis. 87:471-477.

A detached leaf disk assay was developed for screening sour cherry (Prunus cerasus) cultivars for resistance to cherry leaf spot (Blumeriella jaapii). This assay was used to characterize the events occurring in cv. Montmorency (susceptible) and cv. GiSelA 6 [GI 148-1] (resistant) hostpathogen interactions, and to develop a disease scoring scheme to categorize disease severity in sour cherry trees. Forty-three seedlings grown from seeds collected in Russia were screened for resistance using the scoring scheme. Cross infection studies were also carried out with leaf spot isolates from four other Prunus species. These studies were made possible by the development of a new method of culturing $B$. jaapii isolates, using cherry fruit agar. This method enabled large amounts of inoculum to be produced within 2 weeks rather than a couple of months as required previously. In 'Montmorency', disease symptoms were first observed 4 days postinoculation (dpi), with the appearance of small white spots on the undersides of inoculated leaves. These spots gradually grew, producing erumpent spore masses in acervuli approximately 7 dpi. In 'GI 148-1', disease symptoms were also first observed 4 dpi, with the appearance of small transparent lesions. In the majority of cases, these lesions did not increase in size; however, acervuli occasionally were formed approximately 8 dpi. These contained small cirrhi and were often surrounded by an abscission zone. Of the sour cherry seedlings tested, only 'Almaz' open pollinated (o.p.) R1(1) was rated as resistant. 'Almaz' o.p. R1(1) and 'GI 148-1' were also rated as resistant to leaf spot isolates from other Prunus species. These results and the implications for breeding resistant sour cherry cultivars are discussed.
\end{abstract}

Additional keywords: Coccomyces hiemalis, Phloeosporella padi, tart cherry

Michigan is the leading producer of sour cherries (Prunus cerasus L.) in the United States, producing 297 million pounds of sour cherries in 2001 (1). Cherry leaf spot, caused by Blumeriella jaapii (Rehm) Arx (anamorph Phloeosporella padi (Lib.) Arx), is the most important disease of sour cherry in Michigan and throughout the northeastern United States and Canada. All sour cherry cultivars grown in the United States are susceptible to cherry leaf spot, and currently the primary method of control is through the use of fungicides. When not properly managed, cherry leaf spot can cause leaf chlorosis and premature defoliation. Fruit on trees defoliated by cherry leaf spot before harvest show poor coloration, are low in soluble solids, and are softer than fruit on healthy trees (12). Early defoliation can also result in reduced winter hardiness, potentially

Corresponding author: Phillip S. Wharton

E-mail: whartonp@msu.edu

Accepted for publication 7 December 2002.

Publication no. D-2003-0310-03R

(C) 2003 The American Phytopathological Society leading to flower bud loss and tree death $(8,12)$.

A major goal of the Michigan State University (MSU) sour cherry breeding program is the development of new cultivars that have durable resistance to cherry leaf spot. Due to the 25- to 30-year life of a cherry orchard, it is important that the leaf spot resistance bred into new cultivars be effective and durable, reducing the probability of a future breakdown in resistance. Preliminary studies suggested that the interspecific cherry hybrid GiSelA (GI) 148-1 (syn. GI 6) (P. cerasus cv. Schattenmorelle $3 P$. canescens) bred in Germany (18) was resistant to cherry leaf spot and may be a source of resistance (3). The objectives of this study were to develop a rapid detached leaf culture screening assay and characterize the events that occur in susceptible ('Montmorency') and resistant ('GI 148-1') host-pathogen interactions. Such an assay would allow us to maximize the usage of the limited plant material available. Characterization of the events that occur in a resistant host-pathogen interaction allowed us to develop a list of stringent criteria that define resistance. Using these characteristics, 43 seedlings collected in Russia (Table 1) and segregating for resis- tance to cherry leaf spot were screened for potential resistance to cherry leaf spot.

Cross infection studies were also carried out with five isolates of $B$. jaapii from Prunus species growing in Michigan. The use of single-spore isolates in these studies was made possible by the development of a new method of culturing the fungus. Previously, isolates of $B$. jaapii were cultured by point-inoculating a wire-loop scraping of spores and mycelium on Lima bean agar or other similar media $(11,13)$. However, because of the extremely slow growth rate of this fungus on those media, it took up to 3 months to produce enough inoculum for a single inoculation. During this time in culture, the fungus lost its pathogenicity $(11,13)$. Using the new method described here, it was possible to produce enough inoculum within 14 days to inoculate several plants.

\section{MATERIALS AND METHODS}

Culture media. To make cherry fruit agar (CFA), $4 \mathrm{~kg}$ of ripe pitted 'Montmorency' cherries (fresh or frozen) were added to 1 liter of distilled water, brought to a boil, and simmered gently for $2 \mathrm{~h}$. The mixture was then strained through cheesecloth and the resulting extract decanted into 1-liter bottles and autoclaved at $110^{\circ} \mathrm{C}$ for $1 \mathrm{~h}$. For solid media preparation, 300 $\mathrm{ml}$ of cherry fruit extract, $700 \mathrm{ml}$ water, and $20 \mathrm{~g}$ of bacteriological agar were added to a 1-liter flask and autoclaved at $102^{\circ} \mathrm{C}$ for $5 \mathrm{~min}$. The final $\mathrm{pH}$ range of the medium was 3.8 to 4.0 .

Fungal culture. Leaves heavily infected with cherry leaf spot were collected in September 1999 from naturally infected 'Montmorency' sour cherry trees growing at the MSU Clarksville Horticultural Experiment Station (CHES), Clarksville, MI, and stored in paper bags at $-20^{\circ} \mathrm{C}$. These leaves were used as a source of inoculum for all disease screening experiments. For inoculum preparation, the leaves were washed with distilled deionized water, and the resulting spore suspensions were filtered through two layers of cheesecloth and adjusted to $1 \times 10^{6}$ spores $\mathrm{ml}^{-1}$ with a hemacytometer.

For preparation of single-spore isolates for use in cross infection studies, $1-\mathrm{cm}^{2}$ pieces of infected leaves were gently streaked across the surface of petri dishes that contained a thin layer $(5 \mathrm{~mm})$ of po- 
tato dextrose agar and streptomycin at 10 $\mu \mathrm{g} / \mathrm{ml}$ (PDAS), and the plates were incubated at $25^{\circ} \mathrm{C}$ overnight. Single germinated spores were then transferred, with the aid of a dissecting microscope, to fresh petri dishes containing PDAS and incubated under fluorescent lights at $25^{\circ} \mathrm{C}$. Once the isolates had reached $10 \mathrm{~mm}$ in diameter they were transferred to PDA, grown at $25^{\circ} \mathrm{C}$ for 7 days, and stored at $4^{\circ} \mathrm{C}$. For spore production, isolates were transferred to CFA (except the black cherry isolate, which was cultured on PDA) and incubated under fluorescent lights at $25^{\circ} \mathrm{C}$. The isolates were subcultured at 14-day intervals by flooding the surface of the petri dish with sterile distilled deionized water $(5 \mathrm{ml})$ and gently scraping the surface of the media with an L-shaped glass rod to dislodge the spores. Spore suspensions from each culture $(1 \mathrm{ml})$ were then transferred to a new petri dish, spread evenly over the surface of the media using an L-shaped glass rod, and returned to the incubator. For inoculation, the spore suspension was adjusted to $1 \times 10^{6}$ spores $\mathrm{ml}^{-1}$ with a hemacytometer. To prevent loss of pathogenicity of the isolates from growing them in culture too long, host plants were inoculated with the isolates every 2 months and the isolates recovered from the infected tissue.

Whole-plant and branch inoculations. For whole-plant inoculations, spore sus- pensions were prepared as described above and one drop of Tween 20 (approximately $0.01 \% \mathrm{vol} / \mathrm{vol}$ ) was added to the solution. Greenhouse-grown plants (1 to 3 years old) that were actively producing new leaves and had not set terminal bud were sprayed to runoff with the spore suspension and placed in a dew chamber (Percival Scientific, Perry, IA). The inoculated seedlings were incubated in the dark at 18 to $20^{\circ} \mathrm{C}$ for $48 \mathrm{~h}$ with the relative humidity $(\mathrm{RH})$ kept near $100 \%$ so that dew was present on the undersurface of the leaves. After $48 \mathrm{~h}$, plants were further incubated in a 12-h photoperiod for 14 days. The daytime temperature was kept between 19 and $22^{\circ} \mathrm{C}$, with $\mathrm{RH}$ between 60 and $75 \%$, and the nighttime temperature was kept between 18 and $20^{\circ} \mathrm{C}$, with the $\mathrm{RH}$ near $100 \%$ and dew present on the undersurface of the leaves. If sour cherry plants were not available, then branches were cut from actively growing trees (growing at CHES), their cut ends placed in 1-liter bottles of distilled deionized water, and inoculated and incubated as described above. To keep branches fresh, their cut ends were clipped and the water in the bottles was replaced daily for 14 days.

Detached-leaf inoculations. One- to 4day-old leaves were collected from plants that were actively producing new leaves. Leaf age was calculated from the date of

Table 1. Cherry seedlings collected in Russia in 1998 that were screened for leaf spot resistance

\begin{tabular}{lll}
\hline Number of seedlings & Seed parent $^{\mathrm{a}}$ & Collection location $^{\mathrm{a}}$ \\
\hline 8 & Almaz $^{\mathrm{b}}$ & Michurinsk, Russia \\
17 & Prunus fruticosa 18-7-30 & Krymsk, Russia \\
7 & Prunus fruticosa cv. Luganskaya & Krymsk, Russia \\
11 & Prunus fruticosa $18-6-44$ & Krymsk, Russia $^{\mathrm{c}}$ \\
\hline
\end{tabular}

a The seedlings represent open-pollinated progeny from the seed parent.

${ }^{\mathrm{b}}$ Bred in Michurinsk by I. Michurin. Pedigree: $\{[$ P. maackii $\times(P$. fruticosa $\times$ P. cerasus $)] \times($ P. cerasus $\times$ P. avium) $\} \times$ P. cerasus.

${ }^{c}$ Seed collected from Krymsk, but the Krymsk accession is originally from the Volga region.

Table 2. Disease scoring scheme (using a dissecting microscope) used to assess symptom development on sour cherry leaves inoculated with Blumeriella jaapi ${ }^{\mathrm{a}}$

\begin{tabular}{ll}
\hline Score & Symptoms/signs \\
\hline 1 & $\begin{array}{l}\text { Scattered small pigmented lesions } \\
\text { Scattered small pigmented lesions with some larger ones containing sterile aerial } \\
\text { mycelia and/or stunted, bud-like cirrhi in acervuli, often surrounded by an }\end{array}$ \\
& $\begin{array}{l}\text { abscission zone } \\
\text { Similar to } 2 \text { but containing weakly sporulating acervuli with small cirrhi }\end{array}$ \\
3 & 2 to 10 sporulating lesions or lesions covering up to $25 \%$ of the leaf area \\
5 & 11 or more sporulating lesions with lesions covering up to $100 \%$ of the leaf area \\
\hline
\end{tabular}

${ }^{\mathrm{a}}$ Leaves with a disease score of 2 or below were considered resistant, 2 to 3 moderately susceptible, and $>3$ highly susceptible.

Table 3. Origins of the five Michigan Blumeriella jaapii isolates used in the cross infection studies

\begin{tabular}{lll}
\hline Isolate name & Prunus host & \multicolumn{1}{c}{ Origin } \\
\hline Sour cherry & Prunus cerasus & $\begin{array}{c}\text { Clarksville Horticultural Experiment Station } \\
\text { (CHES), Clarksville, Ionia County }\end{array}$ \\
$\begin{array}{l}\text { Sweet cherry } \\
\text { mahaleb }\end{array}$ & $\begin{array}{l}\text { Prunus avium } \\
\text { Prunus mahaleb }\end{array}$ & $\begin{array}{l}\text { CHES Clarksville, Ionia County } \\
\text { Bansing, Ingham County }\end{array}$ \\
$\begin{array}{l}\text { Black cherry } \\
\text { Plum }\end{array}$ & $\begin{array}{l}\text { Prunus serotina } \\
\text { Commercial nursery, Fenville, Allegan County }\end{array}$ \\
\hline
\end{tabular}

unfolding, i.e., when its laminar blades were separated by an angle greater than 90 degrees. Leaves were immersed in $1 \%$ bleach (active ingredient, $5.25 \%$ sodium hypochlorite) for $1.5 \mathrm{~min}$, rinsed for $1 \mathrm{~min}$ in sterile distilled deionized water and then under a stream of sterile distilled deionized water before being dried between two pieces of sterile filter paper. Leaves less than $2 \times 2 \mathrm{~cm}$ were placed abaxial surface up in petri dishes containing $1 \%$ water agar (WA). For leaves larger than $2 \times 2 \mathrm{~cm}$, leaf disks were prepared using a sterilized 1.5$\mathrm{cm}$-diameter cork borer and then placed abaxial surface up in petri dishes containing $1 \%$ WA. Leaves and leaf disks were inoculated by placing a $60-\mu$ droplet of spore suspension $\left(1 \times 10^{6}\right.$ spores $\left.\mathrm{ml}^{-1}\right)$ in the center of the tissue. The petri dishes were then wrapped with Parafilm and incubated for $48 \mathrm{~h}$ in the dark, after which they were maintained in a growth chamber for 14 days in a $12-\mathrm{h}$ photoperiod at 19 to $22^{\circ} \mathrm{C}$. The incubation temperature was kept between 19 and $22^{\circ} \mathrm{C}$, as previous studies have shown that the optimum temperature for leaf spot infection is approximately $20.5^{\circ} \mathrm{C}$ (4). Each leaf disk or leaf was considered a replicate, and 10 leaf disks or leaves were placed in each petri dish. For controls, leaves or leaf disks were treated with sterile distilled deionized water only.

Characterization of disease symptoms. The disease reactions of 'Montmorency' (susceptible) and the interspecific cherry hybrid 'GI 148-1' (resistant) were compared using the detached leaf inoculation. At 1-day intervals up to 14 days postinoculation (dpi), two leaf disks per day were removed from the petri dishes and observed under a dissecting microscope. A severity scale of 1 to 5 (Table 2, Fig. 1) was adapted from the one used by Keitt (11) and developed from the observations of disease symptoms on the 'Montmorency' and 'GI 148-1' leaves. Plants with an average disease score of 2 or less were considered to be resistant, between 2 and 3 moderately susceptible, and above 3 fully susceptible to cherry leaf spot. Only plants with a disease score of 2 or less were considered to be resistant, because in the resistant cv. GI 148-1, lesions with a disease rating of 1 produced no acervuli, while lesions with a disease rating of 2 produced acervuli, but when examined with a dissecting microscope, these acervuli did not appear to contain any sporulating masses. Lesions with a disease rating of 3 or above contained sporulating masses. Any disease symptoms present were noted and recorded on Kodak Elite 100 ASA color slide film. All experiments were replicated three times.

Disease screening of Russian cherry germ plasm. Potentially resistant cherry germ plasm was collected as seeds in Russia in 1998 (Table 1). The seeds were stratified in moist vermiculite and stored at $5^{\circ} \mathrm{C}$ for 5 months. The 43 seedlings ob- 
tained in spring 1999 were grown in a plastic greenhouse at CHES. Disease screening was carried out three times with the following inoculation dates, 21 April, 4 May, and 1 June 2000. At least ten 1- to 4day-old leaves were collected from each of the actively growing seedlings and transported to East Lansing in sealed, inflated plastic bags. Leaves were inoculated using the detached leaf inoculation method and disease symptoms characterized after 14 days, as described above. Leaf disk assays made from 'Montmorency' leaves were used as positive controls to ensure that any resistant reactions observed were not due to poor inoculum, and leaf disks from 'GI 148-1' were used as negative controls. In June 2000, vegetatively propagated cuttings were taken from seedlings that were rated as resistant based on the leaf disk assay. In the spring of 2001, these clones were subjected to further screening using the whole-plant inoculation method described above to confirm their resistance. Two plants of each genotype were inoculated, and 'Montmorency' and 'GI 148-1' plants were used as controls. The experiment was carried out twice (23 April and 8 May 2001). Statistical analyses were carried out using the general linear model (GLM) procedure in the SAS statistical analysis software (SAS Institute, Cary, $\mathrm{NC})$, and treatment means were separated with Fisher's protected LSD $(\alpha=0.05)$. Data from the disease screening experiments carried out in 2000 were analyzed separately from those carried out in 2001.

Cross infection studies with isolates of B. jaapii from Prunus spp. Single-spore isolates of $B$. jaapii were obtained from naturally infected leaves collected from $P$. mahaleb L., $P$. avium L. ('Mazzard' sweet cherry rootstock), $P$. serotina Ehrh. (black cherry), and $P$. domestica L. (plum) plants growing in Michigan (Table 3). Two actively growing plants of $P$. mahaleb, sweet cherry, black cherry, 'Myrobalan' plum ( $P$. cerasifera Ehrh.), sour cherry cultivars Montmorency and GI 148-1, and Almaz o.p. R1(1) were inoculated with each of the five isolates and incubated at 19 to $22^{\circ} \mathrm{C}$ as described above. Plants were scored for disease severity 14 dpi. For experiments with the sour cherry isolate, 'Montmorency' plants were used as controls, and for experiments with the other isolates, plants of their respective hosts were used as controls. Whole-plant experiments with each isolate were carried out twice, and cut branch and leaf disk assays with each isolate were carried out once to confirm the results of the whole-plant inoculations. In whole-plant and cut-branch experiments, disease was evaluated by scoring 10 leaves starting at the tip of the branch (or main stem) and counting leaves backwards along the branch. Experiments with each isolate were carried out separately to avoid crosscontamination between the individual isolates and their respective hosts. Statistical analysis of the data was carried out using $\mathrm{SAS}$ as described above. Because comparison of results from inoculations of whole plant and leaf disks revealed no significant differences, data from the whole plant, leaf disk, and branch inoculations were combined.

\section{RESULTS}

Characterization of disease symptoms in 'Montmorency' (susceptible). There were no visible signs of infection in the susceptible cv. Montmorency for the first 3 dpi. After approximately 4 days, small circular to elliptical white spots (0.5 to 1 $\mathrm{mm}$ in diameter) started to appear on the abaxial surfaces of the inoculated leaves (Fig. 2A). These spots gradually grew (up to $5 \mathrm{~mm}$ in diameter) until approximately 6 dpi, at which time salmon pink raised mounds appeared in the center of the spots (Fig. 2B to D). These mounds increased in height until they ruptured the surface of the leaf, forming acervuli approximately $7 \mathrm{dpi}$ (Fig. 2E). The acervuli contained salmon pink masses of spores that were embedded in a mucilaginous matrix (Fig. 2F and G). The spores exuded from the acervuli as curled cirrhi (Fig. 2F). These were often surrounded by masses of white aerial mycelia (Fig. 2E and G). More than one cirrhus was often formed per acervulus, and they frequently curved inwards and coalesced forming a large mass of spores in the center of the lesion (Fig. 2G). As the acervuli matured, the surrounding white areas gradually turned brown (Fig. 2G). The acervuli were fully mature by approximately $10 \mathrm{dpi}$. In severe infections, the white spots coalesced, often covering the entire area beneath the droplet of spore suspension (Fig. 2H). However, the infection never spread beyond the boundary of the droplet.

Characterization of disease symptoms in 'GI 148-1' (resistant). Disease symptoms in the resistant cv. GI 148-1 also did not become apparent until 4 dpi. Small white circular to elliptical spots present in 'Montmorency' were not formed in 'GI 148-1'. The first symptoms of disease appeared as small, raised, transparent, circular lesions ( 0.5 to $1 \mathrm{~mm}$ in diameter) with a white dot in the center (Fig. 3A). In most cases, the lesion did not increase in size, and by 6 dpi the central white dot had turned red to brown (Fig. 3B to D). Occasionally, the central dot enlarged and by 6 dpi had filled the lesion, leaving a transparent ring around a brownish white spot (Fig. 3E), with acervuli in the center of the lesion by 8 dpi. These acervuli differed from those observed in 'Montmorency' in that they contained stunted cirrhi that formed small buds as they protruded just above the leaf surface (Fig. 3E) and were often surrounded by white aerial mycelia (Fig. 3F) but no erumpent masses of spores (Fig. 3E). In some cases, the acervuli contained only aerial mycelia. In almost all instances where the lesion enlarged and acervuli formed, the transparent ring surrounding the lesion had begun to disintegrate and became an abscission zone by 10 dpi (Fig. 3G). The abscission zone extended around the lesion separating it from the rest of the leaf tissue, and by $14 \mathrm{dpi}$ these acervuli were brown and shriveled (Fig. 3G and H).

Disease screening of Russian cherry germ plasm. Mean disease scores for the susceptible 'Montmorency' and resistant 'GI 148-1' controls were 4.6 and 1.2 , respectively. Only one seedling (Almaz o.p. $\mathrm{R} 1(1)$ ) had a disease rating like that of 'GI 148-1', with a mean disease score of 1.5 (Table 4). However, several others (Almaz o.p. R2(1), Almaz o.p. R11(2), and P. fruticosa 18-6-44 o.p. R19(1)) were moderately susceptible, with mean disease scores of $2.5,2.4$, and 2.9, respectively. All the other plants were highly susceptible, with mean disease scores above 3 . There were significant differences $(P \leq 0.01)$ between individual seedlings in the same disease screening, but there were no significant differences between the same seedling in the different screens. Whole-plant screenings on Almaz o.p. R1(1) plants (carried out in spring 2001), with 'Montmorency' and 'GI 148-1' plants used as controls, confirmed the results of the leaf disk experiments, with Almaz o.p. R1(1), 'Montmorency', and 'GI 148-1' having mean disease scores of $1.1,4.6$, and 1.2 , respectively.

Comparison of the whole-plant and detached-leaf inoculations. When wholeplant versus detached-leaf or leaf-disk inoculations of susceptible 'Montmorency' and resistant 'GI 148-1' and Almaz o.p. R1(1) sour cherry plants were compared, no significant differences in symptom development $(P \leq 0.01)$ were observed at 14 dpi. In preliminary experiments, when inoculated detached leaves or leaf disks were incubated at temperatures above $22^{\circ} \mathrm{C}$, the time between inoculation and appearance of initial disease symptoms was longer (up to 14 days). At temperatures between 25 and $28^{\circ} \mathrm{C}$, infection failed in 'Montmorency' leaf disks (results not shown).

Cross infection studies. In general, cherry leaf spot isolates from $P$. mahaleb, sweet cherry, and sour cherry infected sweet cherry, sour cherry, and P. mahaleb plants but not black cherry and plum (Table 5). The black cherry and plum isolates did not infect sour cherry but were highly virulent on $P$. mahaleb plants and their original host plants. Sweet cherry was not infected by the black cherry isolate and was highly resistant to the plum isolate. In whole plant and branch experiments with the plum isolate, the plum controls were completely defoliated by 14 dpi. Black cherry plants inoculated with the black cherry isolate were not defoliated by 14 dpi, but infection usually covered over 

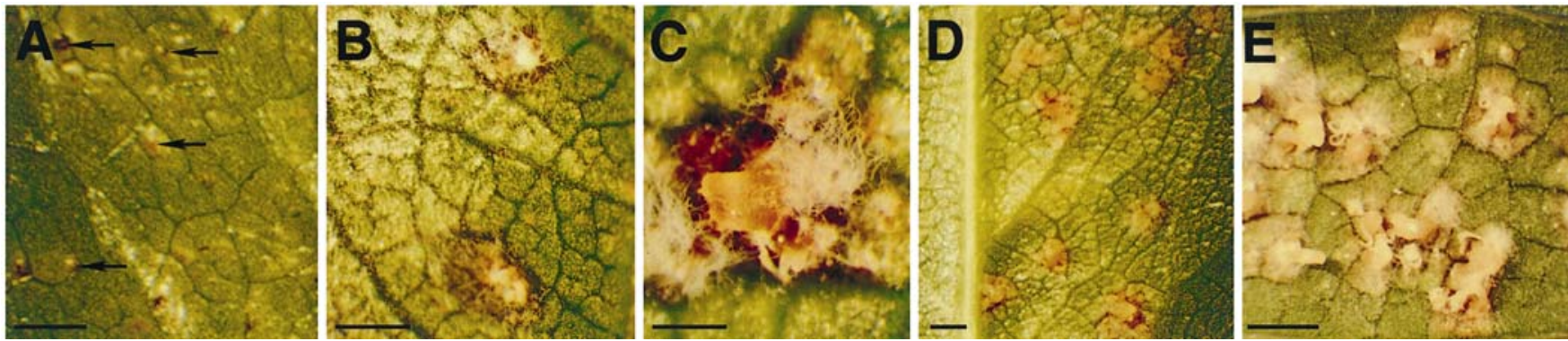

Fig. 1. Characteristic disease symptoms observed after 14 days postinoculation (dpi) and the corresponding disease scores. A, 1 , Scattered small pigmented lesions (arrows) present on leaf. Bar $=1 \mathrm{~mm}$. B, 2, Scattered lesions containing aerial mycelia and/or stunted cirrhi in acervuli, often surrounded by an abscission zone. Bar $=0.5 \mathrm{~mm}$. C, 3, Like (B) but under higher magnification lesions can be seen to contain stunted sporulating cirrhi in acervuli. Bar $=0.25 \mathrm{~mm} . \mathbf{D}, 4,2$ to 10 sporulating lesions present or lesions covering up to $25 \%$ of the inoculated leaf area. Bar $=1 \mathrm{~mm}$. E, 5,11 or more sporulating lesions, with lesions covering up to $100 \%$ of the inoculated leaf area. Bar $=1 \mathrm{~mm}$.
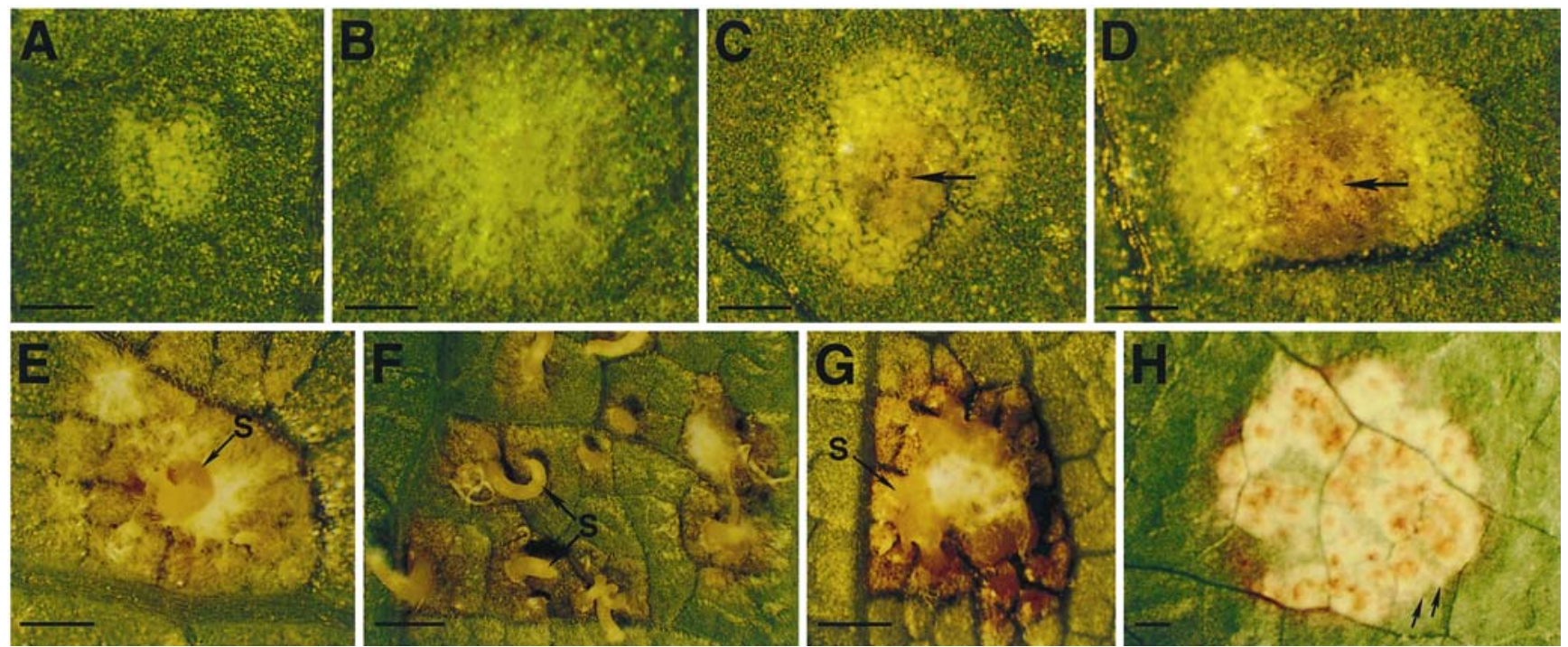

Fig. 2. A chronological composite of the series of events observed during infection of susceptible sour cherry ('Montmorency') leaves by Blumeriella jaapii. A, Small white circular-elliptical spots (0.5-1 mm in diameter) appear on the abaxial leaf surface 4 days postinoculation (dpi). B, These spots grow (up to $5 \mathrm{~mm}$ ) until day 6 , when salmon pink mounds (arrows) appear in the center of the spots $(\mathbf{C}$ and $\mathbf{D})$. A to $\mathbf{D}, \mathrm{Bars}=0.25 \mathrm{~mm}$. E, These mounds increase in height until they rupture the leaf surface forming acervuli approximately 7 dpi. Bar $=0.5 \mathrm{~mm}$. F, The acervuli contain salmon pink masses of conidia embedded in a mucilaginous matrix and are exuded out of the acervuli forming curled tendril-like structures called spore horns $(\mathrm{S})$ or cirrhi. Bar $=$ $1 \mathrm{~mm}$. G, These often coalesce, forming a large mass of spores in the center of the lesion. Bar $=0.5 \mathrm{~mm}$. H, In severe infections, the lesions coalesce covering the entire area beneath the inoculation droplet. Arrows indicate droplet border. Bar $=1 \mathrm{~mm}$.
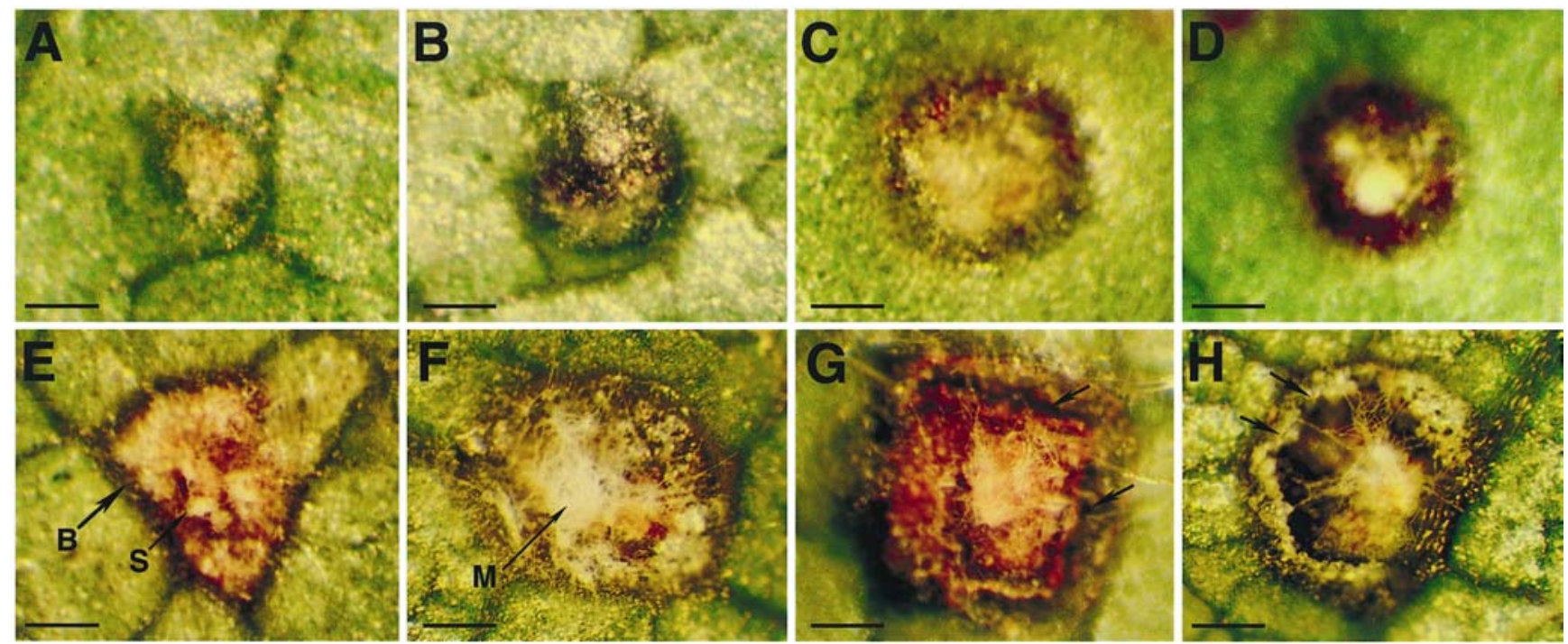

Fig. 3. A chronological composite of the series of events observed during infection of resistant sour cherry leaves ('GI 148-1') by Blumeriella jaapii. A, Small raised transparent circular lesions $(0.5$ to $1 \mathrm{~mm}$ in diameter) containing white centers appear on the abaxial surface of the leaf 4 days postinoculation (dpi). B to D, In most cases the lesion did not increase much in size and by 6 dpi had become pigmented red-brown in color. E, Occasionally the lesions enlarged and by $7 \mathrm{dpi}$ formed acervuli which contained stunted bud-like cirrhi (S) and were brownish white with a transparent border (B). F, These lesions often contained white aerial mycelia (M). The transparent border increased in size by 8 dpi and the lesions started to become more pigmented. G, By $10 \mathrm{dpi}$, the transparent border began to disintegrate forming an abscission zone (arrows) around the lesion. H, By 14 dpi, the abscission zone (arrows) had spread around the whole lesion separating the infected tissue from the rest of the leaf. Bars $=0.25 \mathrm{~mm}$. 
$75 \%$ of the leaf area. Unlike 'Montmorency', 'GI 148-1' and Almaz o.p. R1(1) were rated as resistant to all isolates tested. There were significant differences $(P \leq$ $0.01)$ in relative susceptibility among different selections/species and among isolates. There was also a significant $(P \leq$ 0.01 ) plant by isolate interaction, indicating that the virulence of the isolates differed among selections/species.

\section{DISCUSSION}

The 25- to 30-year life of a cherry orchard requires that the cherry leaf spot resistance bred into new cultivars be effective and durable, reducing the probability that the pathogen will overcome the resistance. Previous investigations (3) sug- gested that the interspecific cherry hybrid 'GI 148-1' was resistant to cherry leaf spot. Our results from the inoculation of wholeplant and detached-leaf/leaf-disk inoculations of 'GI 148-1' under controlled conditions support this observation. Results from the comparison of whole-plant and detached-leaf/leaf-disk inoculations also indicate that the detached-leaf/leaf-disk assay can be used to screen for foliar susceptibility of sour cherry selections to cherry leaf spot. Previous studies have documented the importance of inoculating young leaves (19) and maintaining controlled environmental conditions (4) to achieve successful infection and fungal growth. We also observed that infection was drastically reduced if leaves used in the detached-leaf/leaf-disk assays were more than 5 days old. Therefore, only 1to 4-day-old leaves were used in detached-leaf/leaf-disk assays. Temperature has also been shown to be a limiting factor in the infection of sour cherry leaves by $B$. jaapii (4). The optimum temperature range and $\mathrm{RH}$ for germination and infection of sour cherry leaves by $B$. jaapii conidia have been identified previously as 17.2 to $22^{\circ} \mathrm{C}$ and nearly $100 \%$ RH (4). In preliminary studies, infection of 'Montmorency' leaves failed to occur at temperatures between 25 and $28^{\circ} \mathrm{C}$ along with RH near $100 \%$. Thus for all detached-leaf/leaf-disk inoculations, the incubation temperature was kept between 18 and $22^{\circ} \mathrm{C}$.

Table 4. Disease scores of detached leaves or leaf disks from 43 Russian cherry seedlings and susceptible ('Montmorency') and resistant ('GI 148-1') controls inoculated with Blumeriella jaapii conidia from naturally infected sour cherry leaves in three experiments

\begin{tabular}{|c|c|c|c|c|}
\hline \multirow[b]{2}{*}{ Seedling or clone } & \multicolumn{3}{|c|}{ Disease score $^{a}$} & \multirow[b]{2}{*}{ Mean } \\
\hline & 1 & 2 & 3 & \\
\hline 'Montmorency' & $4.6 \pm 0.5^{b}$ & $4.5 \pm 0.5$ & $4.7 \pm 0.5$ & $4.6 \pm 0.1$ \\
\hline 'GI 148-1' & $1.2 \pm 0.4^{\mathrm{c}}$ & $1.1 \pm 0.3$ & $1.4 \pm 0.5$ & $1.2 \pm 0.1$ \\
\hline Almaz o.p. R1(1) & $1.7 \pm 0.5$ & $1.2 \pm 0.4$ & $1.6 \pm 0.5$ & $1.5 \pm 0.3$ \\
\hline Almaz o.p. R2(1) & $2.7 \pm 0.5$ & $2.2 \pm 0.4$ & $2.6 \pm 0.5$ & $2.5 \pm 0.3$ \\
\hline Almaz o.p. R11(2) & $1.6 \pm 0.7$ & $2.4 \pm 0.5$ & $3.0 \pm 0.0$ & $2.4 \pm 0.7$ \\
\hline Prunus fruticosa 18-6-44 o.p. R19(1) & $2.7 \pm 0.6$ & $3.0 \pm 0.0$ & $3.0 \pm 0.0$ & $2.9 \pm 0.2$ \\
\hline P. fruticosa $18-6-44$ o.p. R4(3) & $3.0 \pm 0.0$ & $3.0 \pm 0.0$ & $3.0 \pm 0.0$ & $3.0 \pm 0$ \\
\hline P. fruticosa $18-7-30$ op. R13(1) & $3.0 \pm 0.0$ & $3.0 \pm 0.0$ & $3.0 \pm 0.0$ & $3.0 \pm 0$ \\
\hline P. fruticosa $18-7-30$ op. R8(3) & $3.2 \pm 0.5$ & $3.1 \pm 0.3$ & $3.0 \pm 0.0$ & $3.1 \pm 0.1$ \\
\hline P. fruticosa cv. Luganskaya op. R5(2) & $3.0 \pm 0.5$ & $3.0 \pm 0.0$ & $3.3 \pm 0.5$ & $3.1 \pm 0.2$ \\
\hline P. fruticosa cv. Luganskaya op. R5(1) & $2.9 \pm 0.0$ & $3.6 \pm 0.7$ & $2.7 \pm 0.5$ & $3.1 \pm 0.5$ \\
\hline P. fruticosa $18-7-30$ op. R8(1) & $3.5 \pm 0.5$ & $2.6 \pm 0.5$ & $3.0 \pm 0.0$ & $3.1 \pm 0.5$ \\
\hline P. fruticosa 18-6-44 o.p. R14(1) & $3.0 \pm 0.0$ & $2.6 \pm 0.5$ & $3.8 \pm 0.4$ & $3.1 \pm 0.6$ \\
\hline P. fruticosa $18-7-30$ op. R8(2) & $2.3 \pm 0.6$ & $3.7 \pm 0.5$ & $3.3 \pm 0.6$ & $3.1 \pm 0.7$ \\
\hline P. fruticosa $18-7-30$ op. R20(2) & $2.4 \pm 0.7$ & $3.0 \pm 0.0$ & $3.8 \pm 0.4$ & $3.1 \pm 0.7$ \\
\hline P. fruticosa $18-6-44$ o.p. R4(1) & $2.3 \pm 0.5$ & $3.0 \pm 0.0$ & $4.0 \pm 0.0$ & $3.1 \pm 0.8$ \\
\hline P. fruticosa 18-6-44 o.p. R3(2) & $3.1 \pm 0.4$ & $3.0 \pm 0.0$ & $3.6 \pm 0.5$ & $3.2 \pm 0.3$ \\
\hline P. fruticosa $18-7-30$ op. R9(1) & $3.9 \pm 0.5$ & $2.4 \pm 0.5$ & $3.3 \pm 0.5$ & $3.2 \pm 0.8$ \\
\hline P. fruticosa $18-7-30$ op. R10(1) & $3.2 \pm 0.4$ & $3.1 \pm 0.4$ & $3.7 \pm 0.5$ & $3.3 \pm 0.3$ \\
\hline P. fruticosa $18-6-44$ o.p. R3(1) & $3.0 \pm 0.0$ & $3.0 \pm 0.0$ & $3.8 \pm 0.5$ & $3.3 \pm 0.4$ \\
\hline P. fruticosa $18-7-30$ op. R20(1) & $2.8 \pm 0.5$ & $3.9 \pm 0.4$ & $3.2 \pm 0.4$ & $3.3 \pm 0.5$ \\
\hline P. fruticosa $18-7-30$ op. R18(2) & $3.3 \pm 0.5$ & $2.8 \pm 0.5$ & $3.9 \pm 0.5$ & $3.3 \pm 0.6$ \\
\hline P. fruticosa $18-7-30$ op. R22(1) & $3.0 \pm 0.0$ & $2.8 \pm 0.4$ & $4.2 \pm 0.8$ & $3.3 \pm 0.8$ \\
\hline P. fruticosa cv. Luganskaya op. R6(1) & $3.3 \pm 0.4$ & $3.2 \pm 0.6$ & $3.7 \pm 0.5$ & $3.4 \pm 0.3$ \\
\hline Almaz o.p. R12(2) & $3.6 \pm 0.4$ & $3.6 \pm 0.5$ & $3.1 \pm 0.5$ & $3.4 \pm 0.3$ \\
\hline P. fruticosa $18-7-30$ op. R21(1) & $3.5 \pm 0.5$ & $3.1 \pm 0.3$ & $3.7 \pm 0.5$ & $3.4 \pm 0.3$ \\
\hline P. fruticosa cv. Luganskaya op. R6(2) & $3.4 \pm 0.5$ & $3.0 \pm 0.0$ & $3.9 \pm 0.3$ & $3.4 \pm 0.4$ \\
\hline P. fruticosa $18-7-30$ op. R9(2) & $3.6 \pm 0.4$ & $3.0 \pm 0.6$ & $3.7 \pm 0.6$ & $3.4 \pm 0.4$ \\
\hline P. fruticosa $18-7-30$ op. R21(2) & $3.1 \pm 0.7$ & $3.3 \pm 0.5$ & $3.8 \pm 0.4$ & $3.4 \pm 0.4$ \\
\hline P. fruticosa cv. Luganskaya op. R7(2) & $3.3 \pm 0.7$ & $3.0 \pm 0.0$ & $4.0 \pm 0.0$ & $3.4 \pm 0.5$ \\
\hline P. fruticosa cv. Luganskaya op. R5(3) & $3.0 \pm 0.0$ & $3.1 \pm 0.3$ & $4.0 \pm 0.0$ & $3.4 \pm 0.6$ \\
\hline P. fruticosa $18-7-30$ op. R18(1) & $3.0 \pm 0.3$ & $3.0 \pm 0.0$ & $4.3 \pm 0.5$ & $3.4 \pm 0.7$ \\
\hline P. fruticosa 18-6-44 o.p. R14(2) & $3.4 \pm 0.5$ & $2.5 \pm 0.5$ & $4.2 \pm 0.4$ & $3.4 \pm 0.8$ \\
\hline P. fruticosa 18-6-44 o.p. R3(3) & $3.6 \pm 0.7$ & $2.2 \pm 0.4$ & $4.3 \pm 0.6$ & $3.4 \pm 1.1$ \\
\hline P. fruticosa $18-7-30$ op. R9(3) & $3.5 \pm 0.5$ & $3.1 \pm 0.4$ & $4.0 \pm 0.0$ & $3.5 \pm 0.4$ \\
\hline P. fruticosa $18-7-30$ op. R19(2) & $3.5 \pm 0.5$ & $3.1 \pm 0.3$ & $4.0 \pm 0.0$ & $3.5 \pm 0.5$ \\
\hline P. fruticosa $18-6-44$ o.p. R4(2) & $3.8 \pm 0.5$ & $3.0 \pm 0.0$ & $4.0 \pm 0.5$ & $3.6 \pm 0.5$ \\
\hline P. fruticosa 18-6-44 o.p. R15(1) & $3.0 \pm 0.7$ & $4.0 \pm 0.0$ & $3.9 \pm 0.6$ & $3.6 \pm 0.5$ \\
\hline Almaz o.p. R12(3) & $3.0 \pm 0.0$ & $3.7 \pm 0.5$ & $4.2 \pm 0.4$ & $3.6 \pm 0.6$ \\
\hline P. fruticosa 18-6-44 o.p. R16(1) & $3.5 \pm 0.8$ & $3.0 \pm 0.0$ & $4.4 \pm 0.5$ & $3.6 \pm 0.7$ \\
\hline Almaz o.p. R11(1) & $3.0 \pm 0.0$ & $4.0 \pm 0.0$ & $4.1 \pm 0.3$ & $3.7 \pm 0.6$ \\
\hline Almaz o.p. R12(1) & $2.8 \pm 0.4$ & $3.9 \pm 0.4$ & $4.5 \pm 0.5$ & $3.7 \pm 0.8$ \\
\hline P. fruticosa $18-6-44$ o.p. R15(2) & $3.6 \pm 0.5$ & $3.0 \pm 0.0$ & $4.6 \pm 0.5$ & $3.7 \pm 0.8$ \\
\hline Almaz o.p. R17(1) & $2.7 \pm 0.5$ & $4.2 \pm 0.4$ & $4.4 \pm 0.5$ & $3.7 \pm 0.9$ \\
\hline P. fruticosa $18-7-30$ op. R7(1) & $3.8 \pm 0.5$ & $3.0 \pm 0.0$ & $4.5 \pm 0.5$ & $3.8 \pm 0.8$ \\
\hline Mean & $3.2 \pm 0.4$ & $3.2 \pm 0.3$ & $3.7 \pm 0.4$ & \\
\hline $\operatorname{LSD}(0.05)$ & 0.4 & 0.3 & 0.3 & \\
\hline
\end{tabular}

a See Table 1 and Figure 1; scores below 2 = fully resistant, between 2 and 3 moderately susceptible, and above 3 fully susceptible.

b Mean \pm standard deviation of 10 replications.

${ }^{c}$ Resistant reactions are in bold type. 
Significant morphological differences were observed between symptom development in susceptible 'Montmorency' and resistant 'GI 148-1' interactions. In susceptible interactions, the circular to elliptical white spots first observed 4 dpi are thought to represent the fungal mycelium growing within the leaf tissue. B. jaapii infects leaves through stomata on the lower leaf surface (10), and the morphology of these white spots is consistent with the fungal mycelia growing inter-cellularly within the leaf tissue. The fact that the tissue around the white spots remains green and appears normal until the formation of acervuli at 7 dpi, suggests that the fungus initially grows biotrophically within the tissue and does not produce any toxins or cell wall degrading enzymes $(17,21)$. Moreover, there are no signs of a host defense response in the leaf tissue until after the formation of acervuli, at which time the tissue surrounding the lesions starts to turn brown. It has been shown that there are enough nutrients present in the apoplast to support the growth of pathogenic fungi (6). Furthermore, it was suggested that the relatively high sugar levels (2 to $10 \mathrm{mM}$ ) initially present during the biotrophic phase may suppress the production of cell wall degrading enzymes with the subsequent switch to necrotrophic growth when nutrients are depleted in the apoplast (6). By initially growing biotrophically within the apoplast, $B$. jaapii may also avoid triggering the host defense response until it has become established within sufficient tissue to lessen the inhibitory effects of defense compounds (16).

Although B. jaapii penetrated the leaf in resistant interactions, it was prevented from proliferating throughout the leaf tissue by a hypersensitive type host defense response thought to be represented by the transparent ring around the white dot. As the hypersensitive response progressed, host defense compounds were produced leading to the pigmentation and in some cases abscission of the tissue surrounding the lesion. Similar pigmentation around hypersensitive lesions has been observed in the host defense responses of sorghum and maize, where the host defense compounds are flavonoid secondary metabolites $(7,14,20)$. Niederleitner et al. (15) found high levels of flavan-3-ols (catechin, epicatechin, and proanthocyanidins) in infected sour cherry leaves as compared to healthy leaves. However, the role of these compounds in the resistance response to infection by $B$. jaapii is questionable since these compounds enhanced spore germination (15). These compounds are also known to be ubiquitous in plants in the Rosaceae and only have weak antifungal activity (5). Thus, it is more likely that these flavan-3-ols are stress metabolites (2) and produced in response to mechanical damage of the tissue surrounding the infection site.

Based on the morphological characteristics observed in susceptible and resistant interactions in response to inoculation with $B$. jaapii, a disease severity index was created to quantify disease severity in sour cherry leaves. A similar disease severity index was used by Keitt (11); however, his index was used to evaluate the presence of susceptible fructifying lesions only. Thus, a disease score of 1 in the Keitt index would be equivalent to a disease score of 3 in our scheme. In the current scheme, the criteria defining resistance were that the plant had to have a mean disease score of 2 or below. In morphological terms, this meant that lesions with a disease rating of 1 produced no acervuli, while lesions with a disease rating of 2 produced acervuli, although these acervuli did not contain sporulating masses. The difference between resistant lesions with a disease rating of 2 and susceptible lesions with a rating of 3 was that lesions with a rating of 3 did produce sporulating masses in small cirrhi. These criteria were particularly stringent to ensure that any lines selected as sources of resistance for use in the breeding program had a high level of resistance.

The disease severity index was used to score 43 Russian seedlings for potential resistance to cherry leaf spot. One resistant seedling, Almaz o.p. R1(1), was identified.
Three other seedlings, Almaz o.p. R2(1), Almaz o.p. R11(2), and P. fruticosa o.p. 18-6-44 o.p. R19(1), had mean disease scores below 3 and showed good resistance to $B$. jaapii. However, they occasionally developed susceptible type lesions and thus are not acceptable for breeding purposes. The resistant seedling Almaz o.p. R1(1) is from a Russian sour cherry cultivar called 'Almaz' that has P. maackii Rupr. in its pedigree. $P$. maackii is known to be resistant to cherry leaf spot $(9,22)$, and the resistance in Almaz o.p. R1(1) may be derived from the $P$. maackii parent. Given that the resistant parent of 'GI 148-1' is probably $P$. canescens, the resistant reactions in 'GI 148-1' and Almaz o.p. R1(1) are probably caused by different genetic mechanisms, even though the morphological manifestations of these resistances appear to be the same. If these two sources of resistance are indeed conferred by different genes, then combining these resistance genes into a resistant cultivar could further enhance the durability of the resistance.

In order to carry out cross infection studies to determine the host range of $B$. jaapii populations in Michigan, it was important to have a defined set of isolates with which to perform inoculations. In the past, such studies have been difficult to carry out because the extremely slow growth rate of B. jaapii in culture made it very difficult to obtain enough inoculum to carry out inoculations. By culturing single spore isolates of B. jaapii on CFA (PDA for the black cherry isolate) and subculturing the isolates by means of spore suspensions, we were able to overcome this problem. This was because culturing spore suspensions encouraged microcyclic conidiation resulting in a massive increase in sporulation of cultures.

The five isolates of $B$. jaapii tested showed significant variation in their virulence toward the six cherry selections and one plum selection tested. Based on the means, standard deviations, and LSD comparisons for the isolates, those from sour cherry, P. mahaleb, and sweet cherry can

Table 5. Mean disease scores of cherry and plum selections inoculated with Blumeriella jaapii conidia from five Prunus species $^{\mathrm{a}}$

\begin{tabular}{|c|c|c|c|c|c|c|}
\hline \multirow[b]{2}{*}{ Prunus plants inoculated } & \multicolumn{5}{|c|}{ Inoculum source or $\boldsymbol{B}$. jaapii isolate } & \multirow[b]{2}{*}{ Mean } \\
\hline & P. mahaleb & $\begin{array}{l}\text { Sweet } \\
\text { cherry }\end{array}$ & $\begin{array}{l}\text { Black } \\
\text { cherry }\end{array}$ & Plum & $\begin{array}{l}\text { 'Montmorency' } \\
\text { sour cherry }\end{array}$ & \\
\hline P. mahaleb & $4.8 \pm 0.4$ & $3.9 \pm 0.4$ & $4.9 \pm 0.4$ & $4.9 \pm 0.3$ & $4.1 \pm 0.3$ & $4.5 \pm 0.5$ \\
\hline Sweet cherry & $3.8 \pm 0.4$ & $4.3 \pm 0.5$ & $0.0 \pm 0.0^{b}$ & $1.0 \pm 0.0$ & $3.3 \pm 0.5$ & $2.5 \pm 1.9$ \\
\hline Black cherry & $0.6 \pm 0.5$ & $\mathrm{~N} / \mathrm{A}^{\mathrm{c}}$ & $5.0 \pm 0.0$ & $0.0 \pm 0.0$ & $0.0 \pm 0.0$ & $1.4 \pm 2.4$ \\
\hline Plum & $0.0 \pm 0.0$ & $0.0 \pm 0.0$ & $0.0 \pm 0.0$ & $5.0 \pm 0.0$ & $0.0 \pm 0.0$ & $1.0 \pm 2.2$ \\
\hline 'Montmorency' & $4.2 \pm 0.4$ & $4.3 \pm 0.5$ & $0.0 \pm 0.0$ & $0.0 \pm 0.0$ & $4.6 \pm 0.5$ & $2.6 \pm 2.4$ \\
\hline 'GI 148-1' & $1.1 \pm 0.3$ & $1.1 \pm 0.3$ & $0.0 \pm 0.0$ & $0.1 \pm 0.3$ & $1.2 \pm 0.4$ & $0.7 \pm 0.6$ \\
\hline Almaz o.p. R1(1) & $1.5 \pm 0.5$ & $1.1 \pm 0.4$ & $0.0 \pm 0.0$ & $0.1 \pm 0.4$ & $1.3 \pm 0.4$ & $0.8 \pm 0.7$ \\
\hline Mean & $2.3 \pm 1.9$ & $2.5 \pm 1.9$ & $1.4 \pm 2.4$ & $1.6 \pm 2.3$ & $2.1 \pm 1.9$ & \\
\hline $\operatorname{LSD}(0.05)$ & 0.4 & 0.4 & 0.0 & 0.3 & 0.4 & \\
\hline
\end{tabular}

a See Table 2 and Figure 1; plants with a score of 0 showed no symptoms of infection, scores of 2 or below = resistant, between 2 and 3 moderately susceptible, and above 3 susceptible. Values are combined means \pm standard deviation of whole-tree, branch, and leaf-disk experiments.

${ }^{\mathrm{b}}$ Resistant reactions (disease score $\leq 2$ ) are in bold type.

c Plant material not available for inoculation. 
clearly be grouped together. Of the three selections susceptible to infection by these isolates, sweet cherry was the most resistant and P. mahaleb was the most susceptible. In addition, $P$. mahaleb was also highly susceptible to infection by the black cherry and plum isolates. Although the black cherry and plum isolates were highly pathogenic, apart from the infection of $P$. mahaleb, they were very host specific. These results agreed well with those from previous investigations $(11,19)$. Keitt (11) and Sjulin et al. (19) both found that sweet cherry was susceptible to infection by cherry leaf spot isolates from sour cherry, but was more resistant to these isolates than sour cherry selections were. Keitt (11) also found that isolates from black cherry and plum were very host specific.

'GI 148-1' and Almaz o.p. R1(1) showed high levels of resistance to all five $B$. jaapii isolates. This is especially important because, although the results suggest that wild black cherry and plum trees would not act as reservoirs of inoculum for the infection of sour cherry orchards, the susceptibility of sour cherry cultivars to $B$. jaapii may differ between regions. Isolates from black cherry and plum from outside Michigan may be more pathogenic on sour cherry than local isolates. Furthermore, isolates of $B$. jaapii from other wild species of cherry such as $P$. pennsylvanica $\mathrm{L}$. f. (pin cherry) and P. virginiana L. (chokecherry) may be pathogenic on sour cherry. Thus, the testing of potentially resistant selections of sour cherry, such as 'GI 1481 ' and 'Almaz' o.p. R1(1), to as many isolates of $B$. jaapii, from as wide a range of Prunus species as possible, is of great importance in ascertaining the extent of their resistance to leaf spot. The broader the spectrum of resistance, the greater the likelihood that the resistance bred into a new cultivar will be durable.

The use of detached-leaf assays and single-spore isolates, as outlined above, is of significant practical value in the identification of resistant sour cherry selections to leaf spot, as it provides a rapid, reliable means of screening large numbers of potentially resistant seedlings. Furthermore, any resistant seedlings identified can then be propagated for use in more detailed disease screening field trials. These methods are now being incorporated into the MSU sour cherry breeding program and used to identify other resistant sources of sour cherry germ plasm.

\section{ACKNOWLEDGMENTS}

This work was funded by a grant from the Michigan Agricultural Experiment Station under Project GREEEN (Generating Research and Extension to meet Economic and Environmental Needs). We thank Oleg S. Zhukov, Ludmila Ishchenko, Adelina Kolesnikova, Elizabeth Jigadlo, and Gennadiy Eremin for providing the Russian sour cherry germ plasm used in this study.

\section{LITERATURE CITED}

1. Anonymous. 2002. USDA-NASS Agricultural statistics 2002. National Agricultural Statistics Service, U.S. Dep. Agric., Washington, DC.

2. Bauer, H., Treutter, D., Schmid, P. P. S., Schmitt, E., and Feucht, W. 1989. Specific accumulation of o-diphenols in stressed leaves of Prunus avium. Phytochemistry 28:13631364.

3. Downey, S. 1999. Genetic diversity of Prunus serotina and the evaluation of other wild species for breeding sour cherry resistance to cherry leaf spot. M.Sc. thesis. Michigan State University, East Lansing.

4. Eisensmith, S. P., and Jones, A. L. 1981. A model for detecting infection periods of $\mathrm{Coc}$ comyces hiemalis on sour cherry. Phytopathology 71:728-732.

5. Grayer, R. J., and Kokubun, T. 2001. Plant fungal interactions: The search for phytoalexins and other antifungal compounds from higher plants. Phytochemistry 56:253-263.

6. Hancock, J. G., and Huisman, O. C. 1981. Nutrient movement in host-pathogen systems. Annu. Rev. Phytopathol. 19:309-331.

7. Hipskind, J., Wood, K., and Nicholson, R. L. 1996. Localized stimulation of anthocyanin accumulation and delineation of pathogen ingress in maize genetically resistant to Bipolaris maydis race $\mathrm{O}$. Physiol. Mol. Plant Pathol. 49:247-256.

8. Howell, G. S., and Stackhouse, S. S. 1973. The effect of defoliation time on acclimation and dehardening in tart cherry (Prunus cerasus L.). J. Am. Soc. Hortic. Sci. 98:132-136.

9. Ishchenko, L. A., Zhukov, O. S., and Shchekotova, L. A. 1979. Coccomyces leaf-blight of sour cherry - Biology of its causal agent and resistance sources. Mikol. Fitopatol. 13(4):327-330.

10. Jones, A. L. 1995. Cherry leaf spot. Pages 2122 in: Compendium of Stone Fruit Diseases. J. M. Ogawa, E. I. Zehr, G. W. Bird, D. F. Ritchie, K. Uriu, and J. K. Uyemoto, eds. American Phytopathological Society, St. Paul, $\mathrm{MN}$.

11. Keitt, G. W. 1918. Inoculation experiments with species of Coccomyces from stone fruit. J. Agric. Res. 13:539-569.

12. Keitt, G. W., Blodgett, E. C., Wilson, E. E., and Magie, R. O. 1937. The epidemiology and control of cherry leaf spot. Univ. Wisc. Agric. Exp. Stn. Res. Bull. 132.

13. Magie, R. O. 1935. Variability of monosporic cultures of Coccomyces hiemalis. Phytopathology 25:131-159.

14. Nicholson, R. L., Jamil, F., Snyder, B. A., Lue, W. L., and Hipskind, J. 1988. Phytoalexin synthesis in the juvenile sorghum leaf. Physiol. Mol. Plant Pathol. 33:271-278.

15. Niederleitner, S., Treutter, D., Zinkernagel, V., and Feucht, W. 1994. Accumulation of flavanols in cherry leaves after infection by the fungus Blumeriella jaapii. Acta Hortic. 318:767-770.

16. O'Connell, R. J., and Bailey, J. A. 1991. Hemibiotrophy in Colletotrichum lindemuthianum. Pages 211-222 in: Electron Microscopy of Plant Pathogens. K. Mendgen and E. D. Lesemann, eds. Springer-Verlag, Berlin.

17. O'Connell, R. J., Bailey, J. A., and Richmond, D. V. 1985. Cytology and physiology of infection of Phaseolus vulgaris by Colletotrichum lindemuthianum. Physiol. Plant Pathol. 27:75-98.

18. Schmidt, H., and Gruppe, W. 1988. Breeding dwarfing rootstocks for sweet cherries. HortScience 23:112-114.

19. Sjulin, T. M., Jones, A. L., and Andersen, R. L. 1989. Expression of partial resistance to cherry leaf spot in cultivars of sweet, sour, duke, and European ground cherry. Plant Dis. 73:56-61.

20. Wharton, P. S., and Julian, A. M. 1996. A cytological study of compatible and incompatible interactions between Sorghum bicolor and Colletotrichum sublineolum. New Phytol. 134(1):24-34

21. Wharton, P. S., Julian, A. M., and O'Connell, R. J. 2001. Ultrastructure of the infection of Sorghum bicolor by Colletotrichum sublineolum. Phytopathology 91:149-158.

22. Zhukov, O. S., Kharitonova, E. N., and Shckekotova, L. A. 1980. Breeding program to obtain high yielding sour cherry varieties resistant to Coccomyces (in Russian). Byul. Nauch. Inform. Tsentr. Genet. Lab. 